\title{
Estudo Ultra-sonográifico, Histerossonográfico, Histeroscópico e Anatomopatológico da Cavidade Uterina de Mulheres na Pós-Menopausa.
}

Autor: Alfeu Cornélio Accorsi Neto

Orientador: Prof. Dr. Wagner José Gonçalves

Dissertação de Mestrado apresentada à Universidade Federal de São Paulo - Escola Paulista de Medicina para obtenção do título de Mestre em Ginecologia em 9 de dezembro de 1999.

No Setor de Climatério da Disciplina de Ginecologia da Faculdade de Medicina de Catanduva e no Setor de Oncocirurgia da Disciplina de Ginecologia da Universidade Federal de São Paulo-Escola Paulista de Medicina, realizou-se o estudo de 58 mulheres na pós-menopausa com vistas à avaliação do endométrio e da cavidade uterina. Efetuaram-se anamnese, exame fisico e ginecológico, e a seguir, realizou-se ultrasonografia transvaginal com medida da espessura do eco endometrial. Em toda paciente, com espessura do eco endometrial maior ou igual a quatro milimetros, procederam-se aos exames de histerossonografia, histeroscopia e biópsia endometrial dirigida. Observaram-se 40 casos $(68,9 \%)$ de alterações endometriais e $18(31,1 \%)$ de endométrio atrófico. Encontram-se pólipo em 30 casos $(51,7 \%)$, hiperplasia sem atipia em 5 $(8,6 \%)$, adenocarcinoma do endométrio em $2(3,4 \%)$, mioma submucoso em $2(3,4 \%)$ e endométrio proliferado em $1(1,8 \%)$ caso. Confrontou-se o resultado dos exames de histerossonografia com o da biópsia da ca- vidade uterina. A histerossonografia detectou 51 $(87,9 \%)$ cavidades com alterações e $7(12,1 \%)$ sem alteração, com sensibilidade de $97,5 \%$ e especificidade de $33,3 \%$. A porcentagem de pacientes com alteração endometrial, quando da histerossonografia anormal, foi de $76,4 \%$ e o endométrio atrófico, quando da histerossonografia normal, foi de $85,78 \%$. Ao confrontarem-se os resultados dos exames de histerossonografia e de histeroscopia, verificou-se boa concordância entre os métodos. Conclui-se que, na avaliação da cavidade uterina de pacientes com eco endometrial maior ou igual a quatro milimetros pela ultra-sonografia transvaginal, a histerossonografia é método capaz de ampliar a visibilização e melhorar o diagnóstico. Pode, ainda, melhorar o processo de triagem pré-operatória para aquelas que precisam de terapêutica com intervenção.

Palavras-chave: Climatério. Endométrio: investigação. Histeroscopia. Histerossonografia. Endométrio: câncer.

\section{Tratamento da Sindrome dos Ovários Policisticos com Metformina: Avaliação de Resultados Clinicos} e Laboratoriais.

Autor: Gustavo Arantes Rosa Maciel

Orientador: Prof. Dr. Edmund Chada Baracat

Dissertação apresentada à Universidade Federal de São Paulo - Escola Paulista de Medicina para obtenção do título de Mestre em Ginecologia em 8 de junho de 2000.

A síndrome dos ovários policísticos (SOP) é uma afecção complexa cujos achados endócrinos principais são o hiperandrogenismo e a anovulação. A insulina tem importante papel na sua fisiopatologia. Para avaliar a eficácia do uso da biguanida metformina no tratamento da SOP foram selecionadas aleatoriamente 29 pacientes obesas e não-obesas, que fizeram uso de metformina $500 \mathrm{mg}$, via oral, de 8 em 8 horas (15) ou placebo na mesma posologia (14) durante seis meses. Foram divididas em 4 grupos e avaliados peso, ciclo menstrual, dosagens de testosterona total e livre, androstenediona e área sob a curva de insulina (ASCI) e glicose (ASCG) antes e após o tratamento. Os resultados mostraram que houve queda significativa de testosterona total $(p<0,05)$ e livre $(p=0,042)$, androstenediona $(\mathrm{p}=0,028)$, ASCG $(\mathrm{p}=0,043)$ e peso $(\mathrm{p}=0,028)$ nas pacientes não-obesas tratadas com metformina. O grupo das obesas tratadas apresentou queda significativa na ASCI $(\mathrm{p}=0,025)$, mas não nos demais parâmetros. Houve melhora nos ciclos menstruais daquelas que fizeram uso da medicação. Concluímos que a metformina mostrou-se eficaz no tratamento do hiperandrogenismo das pacientes não obesas com SOP. No grupo de pacientes obesas houve melhora dos parâmetros avaliados, porém sem significância estatística, após 6 meses de tratamento.

Palavras-chave: Síndrome dos Ovários Policísticos. Resistência à Insulina. Hiperandrogenismo. Obesidade. Ciclo menstrual. 


\section{Sexualidade na Adolescência: Conhecimentos, Atitudes e Práticas dos Adolescentes Estudantes do Municipio de Maceió.}

Autora: Alessandra Plácido Lima Leite

Orientador: Prof. Dr. Rivaldo Mendes Albuquerque

Dissertação apresentada ao Colégio do Curso de Mestrado da Faculdade de Ciências Médicas da Universidade de Pernambuco para obtenção do título de Mestre em Medicina: Área de Concentração em Tocoginecologia, em 29 de dezembro de 2000.

Foi realizado um estudo entre 2.470 adolescentes, estudantes, de 10 a 19 anos, em uma amostra estratificada proporcional de escolas públicas e particulares de Maceió. Utilizou-se um questionário anônimo para investigar os conhecimentos, práticas e atitudes referentes a alguns aspectos da sexualidade. Os resultados mostraram que a grande maioria não sabia qual o período fértil do ciclo menstrual; conhecia ou já havia ouvido falar da sindrome da Imunodeficiência Adquirida; tinha como principais fontes de informação sobre sexo os livros, revistas e jornais, seguidos da televisão e professores da escola e sabia o que era masturbação. Na opinião da maior parte dos adolescentes os pais estimulam mais os filhos homens a ter sexo e reprimem a sexualidade das filhas; homens e mulheres devem portar condom; homens e mulheres dividem a obrigação de evitar a gravidez e a grande maioria não conversa com seus pais sobre sexualidade e anticoncepção. Nesta amostra a menarca ocorreu em média aos 12 anos e a semenarca aos 12,6 anos. A maioria dos rapazes, 60,2\%, e apenas $16,7 \%$ das moças já tinha tido a primeira relação sexual. A iniciação sexual dos rapazes ocorreu em média aos 13,1 anos e das moças aos 14,1 anos. A parceira mais citada pelos rapazes na sexarca foi uma amiga e pelas moças o namorado. Quanto ao uso de anticoncepção, 49,7\% dos rapazes e 36,9\% das moças não usaram nenhum método anticoncepcional na sexarca e, cerca de $1 / 4$ dos adolescentes também não usou na relação sexual mais recente; os métodos anticoncepcionais mais usados na sexarca foram o condom e o coito interrompido e, na relação sexual mais recente, o condom e a pilula. Entre os adolescentes, $86 \%$ dos rapazes e $31,1 \%$ das moças já se masturbou. Através de uma regressão logística, observouse que a probabilidade de iniciar a atividade sexual era maior entre os adolescentes do gênero masculino, sem religião, com idade mais avançada e atrasados em relação à idade escolar. Diante destes resultados, sugere-se a implantação de debates de temas sobre saúde sexual e reprodutiva nas escolas, voltado para alunos, pais e professores, de forma a fornecer subsídios suficientes para diminuir as dúvidas dos adolescentes e preparar os pais e professores para melhor orientar e conviver com este grupo etário.

Palavras-chave: Adolescência. Sexualidade. Contracepção.

\section{Concentração Sérica de Leptina na Sindrome dos Ovários Policisticos: Correlação com Resistência à Insulina e Parâmetros Metabólicos.}

Autora: Hérica Cristina Mendonça

Orientador: Prof. Dr. Rui Alberto Ferriani

Dissertação de Mestrado apresentada à Faculdade de Medicina de Ribeirão Preto da Universidade de São Paulo para obtenção do título de Mestre em Ginecologia, em 10 de abril de 2000.

A descoberta da leptina trouxe novas perspectivas para a relação entre gordura corporal e distúrbios menstruais. Devido aos efeitos da leptina sobre o eixo reprodutivo e sua correlação tanto com a insulinemia quanto com a obesidade, este estudo teve como objetivo testar a possibilidade de que altos niveis de leptina poderiam contribuir na fisiopatologia da Sindrome dos Ovários Policísticos (SOP). Para isso, foram correlacionados os niveis de leptina com a sensibilidade à insulina e com outros parâmetros metabólicos em 28 pacientes com SOP obesas e não-obesas com- paradas com grupo controle de 24 mulheres ovulatórias, também obesas e não-obesas. A leptina, as gonadotrofinas, os androgênios e os lipides foram dosados no jejum e a insulinemia e a glicemia nos intervalos do teste de tolerância oral à glicose -75 g$2 \mathrm{~h}$ (GTT). Foram calculadas as áreas sob as curvas da insulina (ASC-I) e da glicemia (ASC-G), para se definir a resistência à insulina (RI). As pacientes SOP e controles ainda foram subdivididas em: obesas, IMC $\geq 28$ e não obesas, IMC $<28$. Os critérios para o diagnóstico de SOP foram os preconizados pelo NIH (1990). O 
grupo SOP (17-35 anos, IMC 29,4 $\pm 7,8 \mathrm{~kg} / \mathrm{m}^{2}$, média \pm $\mathrm{dp})$ e o grupo controle (16-43 anos, IMC $32,5 \pm 10,6 \mathrm{~kg} /$ $\mathrm{m}^{2}$ ) foram semelhantes. Os níveis de leptina no grupo SOP se correlacionaram significativamente com o IMC $(\mathrm{r}=0,74 ; \mathrm{p}<0,0001)$ e com o estradiol $(\mathrm{r}=0,48 ; \mathrm{p}<0,008)$; Houve ainda uma tendência à correlação da leptina com o ICQ (indice cintura/quadril) e com a ASC-I $(\mathrm{r}=0,36 ; \mathrm{p}=0,05)$. Os níveis séricos de leptina (média \pm dp) não foram maiores no grupo SOP que no controle (20,5 e 27,3 $\mathrm{ng} / \mathrm{ml}$ respetivamente). As obesas, independentemente se SOP ou controles, apresentaram niveis significativamente maiores de leptina (32,2 ng/ $\mathrm{ml})$ que as não-obesas $(13,5 \mathrm{ng} / \mathrm{ml})$. Estes resultados indicam que as concentrações séricas de Leptina nas portadoras da SOP estão fortemente relacionados ao IMC e não são afetados independentemente pela insulinemia ou pela hiperandrogenemia. Entretanto, o estradiol pode ter um papel regulador da produção de Leptina na SOP.

Palavras-chave: SOP. Leptina. Resistência à insulina. Hiperandrogenismo. Obesidade.

\section{$\operatorname{RBCO} 23(02): 125,2001$}

\section{Tratamento de Ratas Diabéticas Prenhes com Extrato de Bauhinia forficata (pata-de-vaca): Repercussões Materno-fetais.}

Autora: Débora Cristina Damasceno

Orientadora: Profa. Dra. Marilza Vieira Cunha Rudge

Tese apresentada ao Programa de Pós-Graduação em Ginecologia e Obstetrícia da Faculdade de Medicina de Botucatu - Unesp- para obtenção do título de Doutor, em 15 de dezembro 2000.

Introdução: a hiperglicemia materna aumenta a produção de radicais livres que estão relacionados com complicações diabéticas e os antioxidantes exógenos têm importância na profilaxia de embriopatias diabéticas. Objetivos: estudar a influência do diabete grave no sistema antioxidante de ratas prenhes e a ação do extrato de Bauhinia forficata no controle materno da glicemia, lipides, colesterol, triglicérides, proteínas totais, sistema antioxidante, no peso fetal e placentário e na redução de embriopatias.

Material e Método: ratas Wistar prenhes divididas em 4 grupos: não-diabético e diabético controles; não-diabético e diabético tratados com extrato aquoso de Bauhinia forficata. O diabete foi induzido antes da prenhez pela injeção intravenosa de streptozotocin $(40 \mathrm{mg} /$ $\mathrm{kg}$ de peso). A administração do extrato da planta ou da água destilada (controle) foi por via oral (gavage) em 3 doses crescentes: $500 \mathrm{mg} / \mathrm{kg}$ do 0 ao $4^{\circ}$ dia de pre-

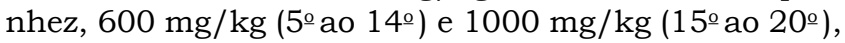
sendo dois terços da dose pela manhã e um terço à tarde, com ajuste diário da dose conforme peso do animal. No 21ํ dia de prenhez, após laparotomia, foi feito esgotamento da volemia materna e retirada do figado para dosagens bioquímicas. Os recém-nascidos (RN) foram retirados, pesados e analisados quanto à presença de malformações e anomalias externas e internas.

Resultado: o tratamento com $B$. forficata não controlou a glicemia, hiperlipemia, hipercolesterolemia, hipertrigliceridemia de ratas diabéticas, nem adequou o maior número de $\mathrm{RN}$ pequenos para idade de prenhez. Aumentou a atividade de glutationa reduzida (GSH) e o depósito de glicogênio hepático e diminuiu a incidência de malformações e anomalias viscerais. Conclusão: os resultados sugerem que a redução das malformações e anomalias viscerais está relacionada com a tendência a redução do estresse oxidativo.

Palavras-chave: Diabete melito. Complicações da gravidez. Extratos vegetais.

\section{Correlação entre os Achados Mamográficos, Ultrassonográficos e Histopatológicos de Lesões não-} palpáveis de Mama.

Autor: Luciano Antonio Ribeiro Spina

Orientador: Prof. Dr. Juradyr Moreira de Andrade

Dissertação de Mestrado apresentada a Área de Tocoginecologia do Departamento de Ginecologia da Faculdade de Medicina de Ribeirão Preto da Universidade de São Paulo em 25 em fevereiro de 2000.

Objetivos: correlacionar as alterações mamárias detectadas nas mamografias com as alterações visualizadas através do ultra-som mamário e os diagnósticos histopatológicos. Analisar a eficiência do exame 
ultrasonográfico para a localização nas lesões não palpáveis de mama.

Pacientes e Métodos: foram incluídas neste estudo 367 pacientes com lesões não-palpáveis de mama. Estas pacientes foram submetidas à seleção de acordo com critérios definidos previamente, permanecendo 64 pacientes com lesões suspeitas e estas foram investigadas através de biópsias excisionais. Todas as pacientes foram submetidas a mamografia bilateral e seguidas de exame ultra-sonográfico, isto é, toda lesão visível ao ultra-som era localizada por meio deste. Caso não fosse possivel, a localização das lesões era feita por estereotaxia. As pacientes foram divididas em dois grupos: A (localização por agulhamento por estereotaxia) e B (localização da lesão pelo ultra-som). Resultados: no grupo A foram incluidas 27 pacientes $(42,2 \%)$ que foram divididas conforme as seguintes patologias: 6 pacientes $(22,2 \%)$ com lesões nãoproliferativas, 14 pacientes com lesões proliferativas sem atipias $(51,8 \%), 3$ pacientes com lesões proliferativas com atipias $(11,1 \%)$ e 4 pacientes com carcinoma intraductal ou invasor $(14,9 \%)$. No grupo B foram incluídas 37 pacientes $(57,8 \%)$, entre as quais foram observados os seguintes resultados histopatológicos: 13 pacientes com lesões não proliferativas $(35,1 \%), 19$ pacientes com lesões proliferativas sem atipias $(51,3 \%), 2$ pacientes com lesões proliferativas com atipias $(5,4 \%)$ e 3 pacientes com lesões neoplásicas (8,2\%). Não houve diferença quanto a distribuição das lesões em função do método de localização empregado.

Conclusão: o método preferencial para marcação das lesões foi a ultra-sonografia, isto é, foi possivel localizar as lesões de mama não-palpáveis em 92,5\% dos procedimentos, deixando apenas 3 procedimentos $(7,5 \%)$ em dúvida quanto a sua localização, demonstrando ser o ultra-som eficiente, rápido e de fácil execução. No entanto, a correlação entre a mamografia com a ultra-sonografia mostrou maior acurácia da mamografia para a detecção e lesões não-palpáveis de mama. Apesar dos resultados histológicos isolados serem muito próximos para ambos os métodos, a estereotaxia foi mais precisa no diagnóstico das lesões neoplásicas.

Palavras-chave: Mama: câncer-diagnóstico. Mamografia. Lesões não-palpáveis da mama.

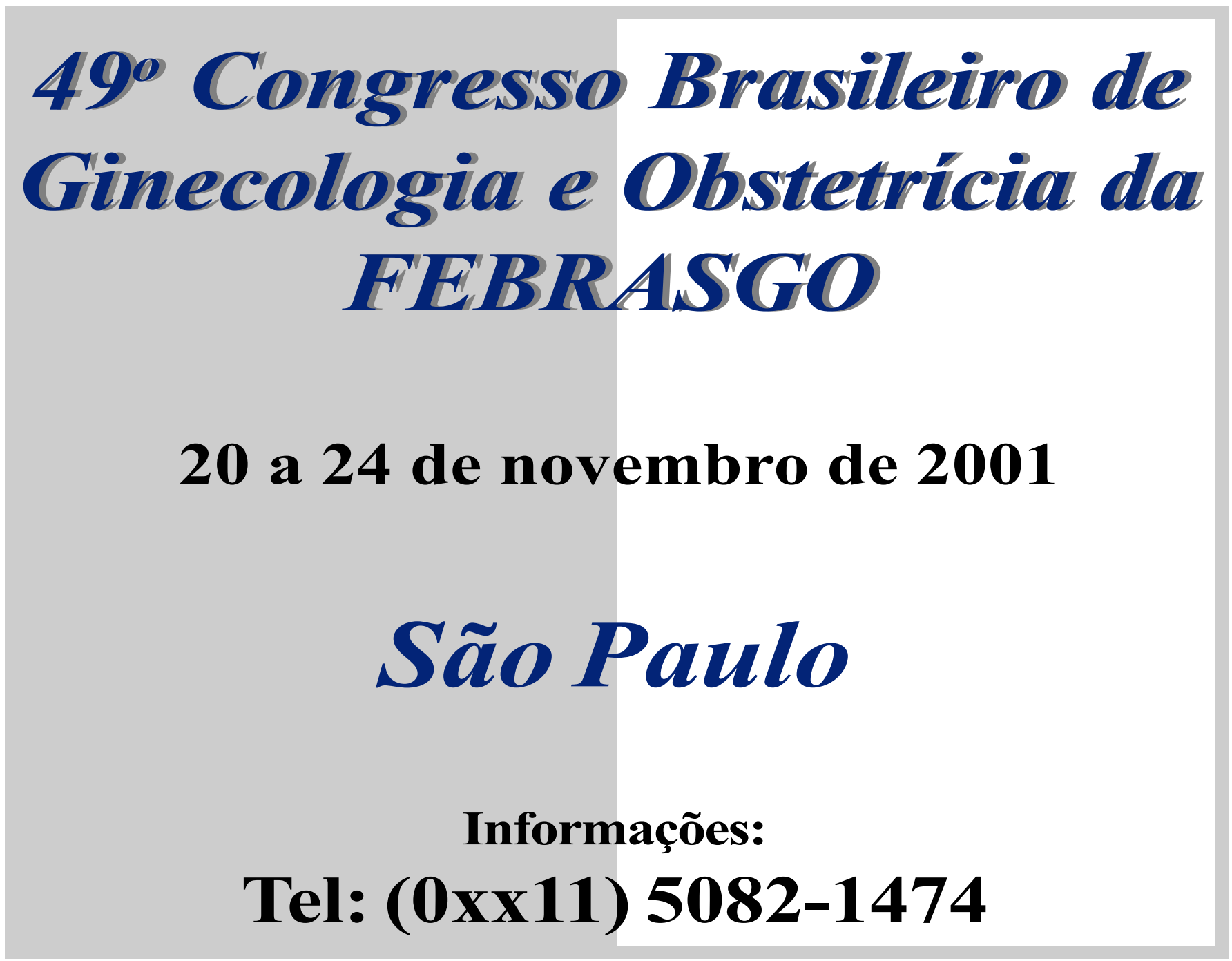

